\title{
ATENÇÃO FARMACÊUTICA NA MENOPAUSA
}

\author{
BASTIANI, Jaqueline de ${ }^{1 *}$; MIGUEL, Marilis D2. , ZANIN, Sandra M. W. ${ }^{3}$ \\ RECEBIDO: ABRIL/2005 ACEITO: JUNHO/2005 \\ 1- Farmacêutica Bioquímica (UFPR) Parte da Monografia de Especialização em Farmácia Magistral \\ (CBES-PR). \\ 2-Prof. Adjunto de Farmacotécnica I , Curso de Farmácia UFPR, Orientadora \\ 3-Prof. Adjunto de Farmacotécnica II, Curso de Farmácia UFPR, Co- orientadora \\ *email:jaquedb@bol.com.br
}

\begin{abstract}
RESUMO:
Análise de uma pesquisa participativa dentro de uma abordagem qualitativa, realizada no período de abril a agosto de 2004, em uma farmácia magistral na cidade de Curitiba tendo como público alvo da pesquisa, mulheres que se encontram no período da menopausa. Os resultados obtidos com a aplicação de um instrumento de avaliação ao grupo no que se refere à isoflavona determina o alto índice de prescrição do fitoestrógeno, as especialidades médicas que a prescrevem, o intervalo de dosagem prescrita e as associações mais freqüentes. O trabalho também retrata a análise das formulações quanto à forma farmacêutica prescrita, a incidência dos sintomas mais freqüentes na menopausa, a incidência de doenças associadas e a incidência de cuidados especiais (hidratação cutânea, proteção solar, nutrição , prática de exercícios, tabagismo). O resultado dessa pesquisa participativa permite definir a amplitude da menopausa no que diz respeito ao tratamento, à sintomatologia, às doenças associadas e à Atenção Farmacêutica.
\end{abstract}

Palavras-chave: menopausa, atenção farmacêutica, isoflavona, farmácia magistral.

\section{ABSTRACT:}

Analysis of a participative investigation within a qualitative approach, carried out in the peiod of april through august of 2004, in a magistral pharmacy in the city of Curitiba, having as target audience for the investigation, women undergoing menopause. The results obtained with the application of an evaluation instrument on the group, relating to isoflavona as determining the high index of prescription of the phytoestrogen, the medical specialties that prescribe it, the interval of dosage prescribed and the most common associations. The work also portrays the analyses of formulations as to the pharmaceutical form prescribed, the incidence of special care (cutaneous hydration, solar protection, nutrition, exercise, use of tobacco). The result of this participative investigation allows the definition of the amplitude of menopause in relation to th treatment, to the symptoms, to associated illnesses and to pharmaceutical attention.

Key words: menopause, pharmaceutical care, isoflavona, magistral pharmacy.

\section{INTRODUÇÃO}

A Atenção Farmacêutica conforme (PAULOS, 2002), é a prática farmacêutica onde o profissional assume responsabilidades com relação ao resultado clínico dos Pacientes, caracteriza-se por uma interação direta entre o paciente e o farmacêutico, com a finalidade de que este último satisfaça as necessidades relacionadas com o uso de medicamento pelos pacientes. Dessa maneira o farmacêutico colabora amplamente com os demais profissionais de saúde para melhorar a qualidade de vida dos pacientes, assumindo papel de sujeito co-responsável na terapêutica do paciente. Essa prática visa o respeito ao ser humano, a responsabilidade ética e o conhecimento técnico e 
científico garantido pelo âmbito profissional.

Numa farmácia de manipulação, de acordo com (SOUZA, 2002), existem várias atividades exercidas pelo farmacêutico que requerem responsabilidade técnica, dentre estas destacam-se a manipulação e a dispensação, igualmente importantes e necessárias, pois envolvem a execução de fórmulas e o serviço de informação e orientação à população sobre o uso correto do medicamento. Ambas atividades perpassam a responsabilidade técnica-científica e o medicamento manipulado dentro dos requisitos técnicos exigidos não é garantia de resultados efetivos e definitivos na farmacoterapia preconizada pelo médico, a orientação do profissional farmacêutico se faz necessária na informação, educação e aconselhamento dos clientes sobre os medicamentos que dispensa.

O serviço de Atenção Farmacêutica pode ser aplicado a grupos específicos de pacientes, sendo considerado neste trabalho o grupo de mulheres que se encontram no período da menopausa. Atualmente, com o prolongamento da expectativa de vida, os períodos menopáusicos e pós-menopáusicos estão se tornando cada vez mais significativos na vida da mulher.

O uso de fitoestrógenos apresenta vantagens para a menopausa, de acordo com (CIAPAUCH et al, 2002), os fitoestrógenos encontrados em várias plantas comestíveis podem ter efeitos estrogênicos e anitestrogênicos. Estudos epidemiológicos, comparando população asiática versus ocidental, tem sido interpretados no sentido de que uma dieta rica em fitoestrógenos melhoraria os sintomas da menopausa e protegeria contra câncer de mama, perda óssea e doenças cardiovasculares.

\section{MATERIALE MÉTODOS}

O referido trabalho compõe uma pesquisa participativa dentro de uma abordagem qualitativa realizada numa farmácia de manipulação de médio porte, situada no bairro Batel na cidade de CuritibaPr, no período de abril a agosto de $2004 \mathrm{e}$ teve como público alvo mulheres da classe média alta que se encontram no período da menopausa.

Realizou-se uma pesquisa participativa de abordagem qualitativa, onde aplicou-se um instrumento de avaliação a 60 mulheres com idade média de 52 anos que se encontram no período da menopausa e analisou-se as respectivas prescrições.

O instrumento de avaliação permitiu coletar informações sobre dados pessoais, medicamentos (associações), doenças associadas à menopausa, sintomas na menopausa, cuidados especiais (uso de filtro solar, hidratante, exercícios físicos, acompanhamento nutricional, tabagismo), anticoncepção, periodicidade da realização de exames laboratoriais e dúvidas das mulheres menopausadas.

As prescrições foram analisadas quanto às associações, forma farmacêutica prescrita, especialidade médica quanto à prescrição da isoflavona e dosagem de isoflavona prescrita.

A tabulação e a análise e interpretação dos dados foram realizados segundo SAMARA e BARROS (1998), de modo uniforme dentro dos parâmetros qualitativos e os resultados foram apresentados em porcentagem, sintetizados na forma de gráficos ( do tipo linear, colunas e setores) e tabela. 


\section{RESULTADOS E DISCUSSÃO}

\subsection{QUANTO AO MEDICAMENTO UTILIZADO}

Como representa a FIGURA 1 a isoflavona é utilizada por $74 \%$ das mulheres, numa concentração que variou de $10 \mathrm{mg}$ a $160 \mathrm{mg}$ (FIGURA4). A tibolona é utilizada por $11 \%$ das mulheres, os estrógenos conjugados e a metiltestosterona por $6 \%$ das mulheres, a progesterona e o propionato de testosterona por $3 \%$ das mulheres.

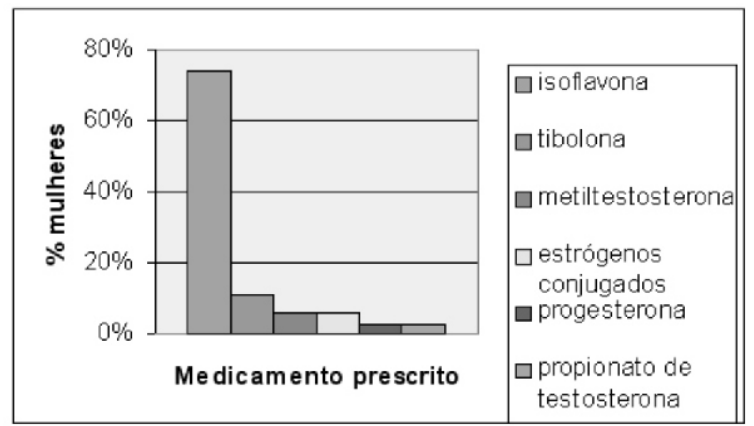

\section{FIGURA 1 - MEDICAMENTOS UTILIZADOS PELAS MULHERES DO GRUPO}

O alto índice do uso da isoflavona pode ser atribuído ao grande apelo publicitário feito aos produtos derivados da soja, a qual conforme DELCIO (2003), possui fitoestrógenos capazes de atenuar os sintomas da menopausa. Também devese considerar que as mulheres com histórico familiar para câncer de mama e de útero são orientadas pelo médico para o uso da isoflavona, já que neste caso a terapia de reposição hormonal convencional não é bem aceita.

\subsection{QUANTO À FORMA FARMACÊUTICA PRESCRITA}

As cápsulas correspondem a $89 \%$ das fórmulas prescritas e as formas farmacêuticas creme ou gel totalizam 11\%, conforme FIGURA2.

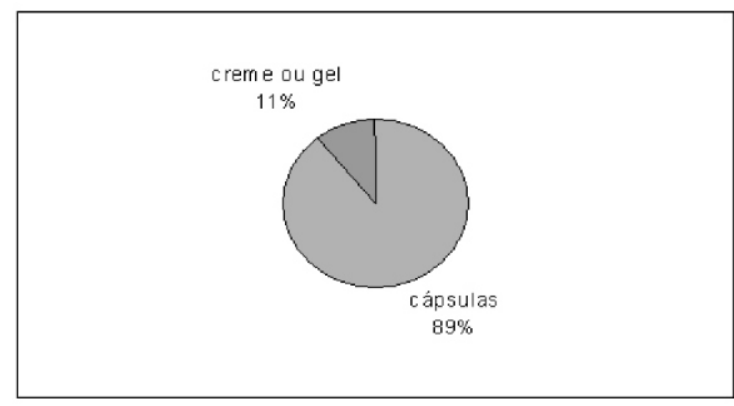

\section{FIGURA 2 - FORMA FARMACÊUTICA DOS MEDICAMENTOS PRESCRITOS}

Para as formulações de uso oral (cápsulas) predominou o uso de isoflavona, seguido de tibolona e metiltestosterona. As formulações de uso tópico (creme ou gel) tinham como ativos os estrógenos conjugados e o propionato de testosterona. 


\subsection{QUANTO ÀS ESPECIALIDADES MÉDICAS}

De acordo com a FIGURA 3, os médicos dermatologistas foram responsáveis por $69 \%$ das prescrições da isoflavona, os ginecologistas por $6 \%$, os endocrinologistas por $4 \%$, os clínicos gerais por $19 \%$ e os cardiologistas por $2 \%$.

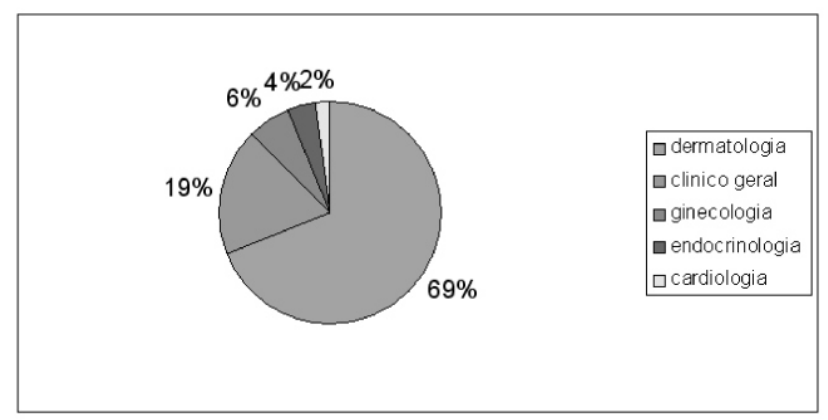

\section{FIGURA 3 - ESPECIALIDADE CLÍNICA PRESCRITORA DA ISOFLAVONA}

De acordo com ALVES e SILVA (2003), a isoflavona possui ação antioxidante a qual previne o envelhecimento e melhora o aspecto da pele, o que pode explicar o resultado encontrado para a especialidade dermatologia (69\%), no entanto, a presença de fitoestrógenos conforme DELCIO (2003), mostra efeitos sistêmicos da isoflavona para a atenuação dos sintomas da menopausa.

Os ginecologistas foram responsáveis por uma pequena parcela (6\%), muitos deles resistem à prescrição da isoflavona, por desacreditar na ação da mesma e preferir o tratamento de reposição hormonal convencional.

Cerca de $19 \%$ das prescrições foram realizadas por médicos clínicos gerais, isso demonstra, que muitas mulheres não encaram a menopausa como uma síndrome que necessita de um tratamento médico ginecológico e/ou endocrinológico, e cabe ao farmacêutico através do exercício da Atenção Farmacêutica, orientar essas mulheres para um tratamento médico especializado, na medida que detecta essa necessidade através dos relatos da sintomatologia.

\subsection{QUANTOADOSAGEM DA ISOFLAVONAPRESCRITA}

Como mostra a FIGURA 4, a dosagem variou de $10 \mathrm{mg}$ a $160 \mathrm{mg}$, sendo a dosagem de $50 \mathrm{mg}$ e $80 \mathrm{mg}$, respectivamente responsáveis por $33 \%$ e $23 \%$.

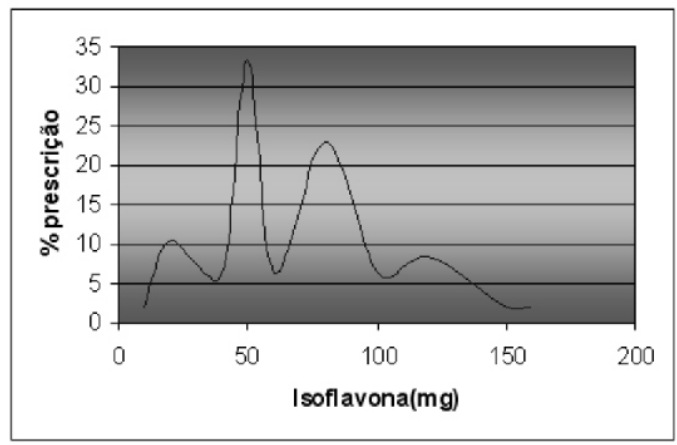




\section{FIGURA 4 - INTERVALO DA DOSAGEM DA ISOFLAVONA PRESCRITA}

Existe uma grande dificuldade para a pesquisa da dosagem da isoflavona em literaturas clássicas, restrigindo-se na maioria, aos ensaios publicados através de literatura contemporânea. A falta de fontes que definam a dosagem da isoflavona permite que os médicos prescrevam um grande intervalo, porém, esta amplitude pode estar comprometendo o efeito terapêutico.

\subsection{QUANTO ÀS ASSOCIAÇÕES MAIS FREQUENTES}

Nas associações, $53 \%$ das mulheres usam isoflavona associada à vitamina $\mathrm{C}$, $49 \%$ ao licopeno, $16 \%$ à fluoxetina, $9 \%$ ao ginkgo biloba, $9 \%$ ao colágeno, $5 \%$ ao betacaroteno e $5 \%$ à levotiroxina, como mostra a FIGURA 5

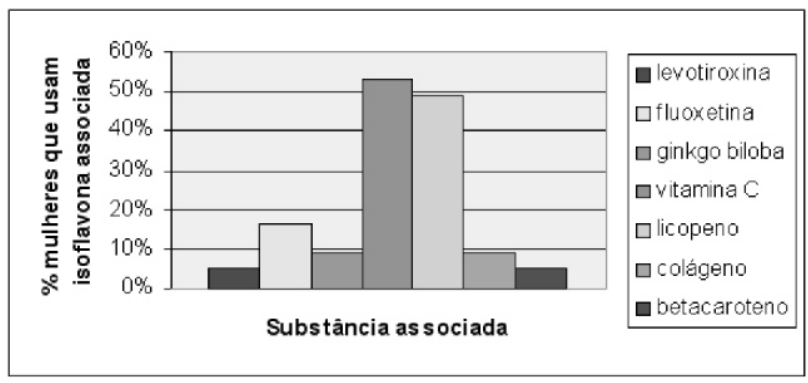

FIGURA 5 -ASSOCIAÇÕES MAIS FREQUENTES ENCONTRADAS NO GRUPO

A isoflavona prescrita pelos dermatologistas foi associada com substâncias antioxidantes (vitamina C e licopeno), buscando dessa maneira, aumentar a ação anti radicais livres da formulação.

Como mostra a FIGURA 6 doenças da tireóide e depressão estão associadas ao período da menopausa, o que pode explicar o uso de tiroxina e fluoxetina associadas à isoflavona. MENOPAUSA

3.6 QUANTO ÀS PATOLOGIAS ASSOCIADAS AO PERÍODO DA

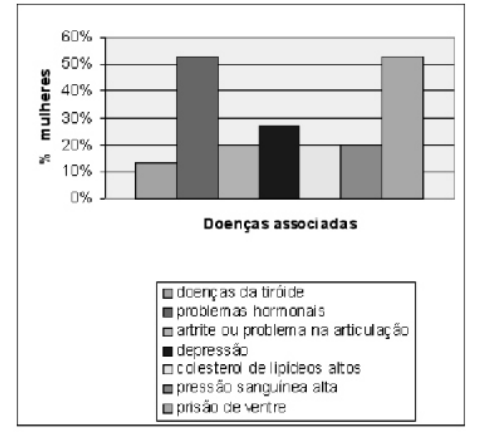

FIGURA 6 - DOENÇAS ASSOCIADAS À MENOPAUSA 
No grupo estudado, $53 \%$ das mulheres possuem problemas hormonais, $53 \%$ tem prisão de ventre, $27 \%$ tem depressão, $20 \%$ tem artrite ou problemas nas articulações, $20 \%$ tem colesterol de lipídeos altos, $20 \%$ tem pressão sanguínea alta (considerando-se os valores utilizados pela Organização Mundial de Saúde (OMS) $160 \mathrm{mmHg}$ de pressão sistólica e/ou $95 \mathrm{mmHg}$ de pressão diastólica) e $13 \%$ tem doenças da tireóide, conforme representado na FIGURA6.

O período da menopausa coincide alterações hormonais com modificações fisiológicas decorrentes da idade, várias doenças e distúrbios podem acometer simultaneamente a saúde da mulher, existindo a necessidade de um acompanhamento farmacêutico diferenciado para a farmacoterapia. O uso de medicamentos associados resultante da existência de duas ou mais doenças simultâneas, que muitas vezes são tratadas por médicos diferentes, pode representar incompatibilidades medicamentosas e colocar em risco a farmacoterapia. A Atenção Farmacêutica detecta essas incompatibilidades e através de um contato interdisciplinar, modificações podem ser feitas a fim de garantir um tratamento adequado.

\subsection{QUANTO AOS SINTOMAS DA MENOPAUSA}

TABELA 1- SINTOMAS APRESENTADOS PELAS MULHERES DO GRUPO

\begin{tabular}{|l|c|}
\hline SINTOMAS & \% MULHERES \\
\hline Ansiedade & 67 \\
\hline Distúrbios do sono & 67 \\
\hline Ganho de peso & 53 \\
\hline Calores & 53 \\
\hline Secura vaginal & 53 \\
\hline Sudorese noturna & 47 \\
\hline Diminuição do desejo sexual & 47 \\
\hline Dores musculares & 40 \\
\hline Dores de cabeça & 33 \\
\hline Mudanças de humor & 33 \\
\hline Pele/ cabelo secos & 33 \\
\hline Dor no quadril & 20 \\
\hline Bexiga baixa & 20 \\
\hline Irritabilidade & 20 \\
\hline Menstruação irregular & 17 \\
\hline Urina solta & 13 \\
\hline
\end{tabular}


O resultado mostra que as mulheres do grupo possuem multiplicidade de sintomas e concorda com MARTINS (1996), que demonstra que em $75 \%$ das mulheres, a menopausa é acompanhada de múltiplos sintomas relacionados com a modificação dos níveis hormonais e a sintomatologia pode ser esquematizada em quatro grupos: alterações psíquicas; alterações provenientes de distúrbios nervosos e dos vasos sanguíneos; alterações do aparelho geniturinário e alterações da musculatura, da pele e do esqueleto ósseo.

\subsection{CUIDADOS COM A PELE, NUTRIÇÃO, EXERCÍCIOS FÍSICOS E TABAGISMO}

No grupo $80 \%$ das mulheres praticam exercícios físicos, $80 \%$ fazem uso de hidratante facial e/ou corporal, $60 \%$ fazem uso de filtro solar, $40 \%$ possuem dieta balanceada e/ou enriquecida com fitoestrógenos da soja, $20 \%$ já fizeram acompanhamento com nutricionista e $20 \%$ é fumante.

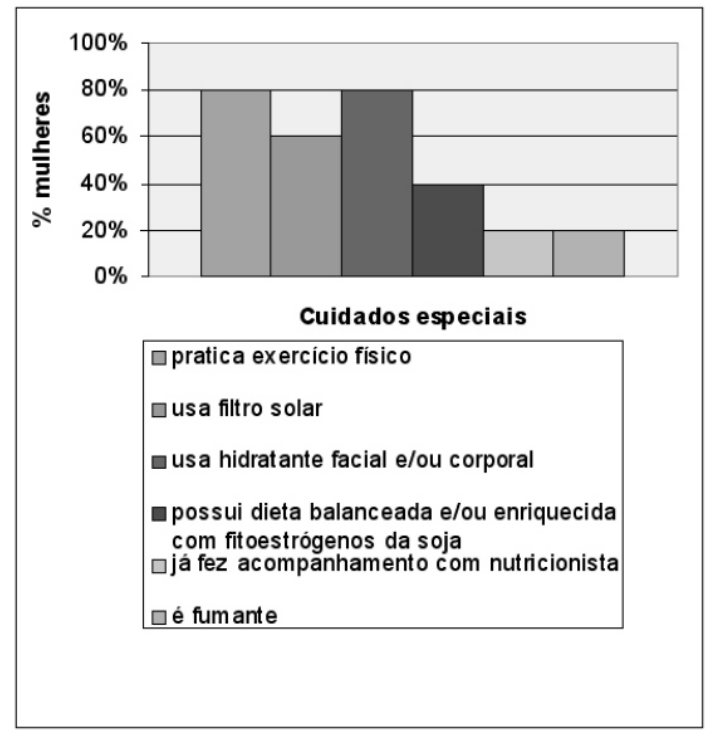

\section{FIGURA 7 - CUIDADOS COM A PELE, CORPO, NUTRIÇÃO E SAÚDE}

A Atenção Farmacêutica mostra-se importante na prevenção de doenças quanto aos cuidados especiais. A orientação para o uso de fotoprotetores pode prevenir o câncer de pele e o aparecimento de manchas; a orientação para a realização de exercícios físicos e para o acompanhamento nutricional pode prevenir a obstipação intestinal, as doenças cardiovasculares, a obesidade; o alerta e conscientização para os riscos do tabagismo pode prevenir o câncer de pulmão, se tornando útil dessa maneira o serviço de Atenção Farmacêutica para a prevenção de doenças.

\subsection{QUANTO À DIETA ALIMENTAR}

Representados na FIGURA 8, 40\% das mulheres tomam leite de soja, $20 \%$ possuem dieta rica em fibras, $20 \%$ usam linhaça, $13 \%$ usam aveia e $13 \%$ tomam vitamina $\mathrm{E}$. 


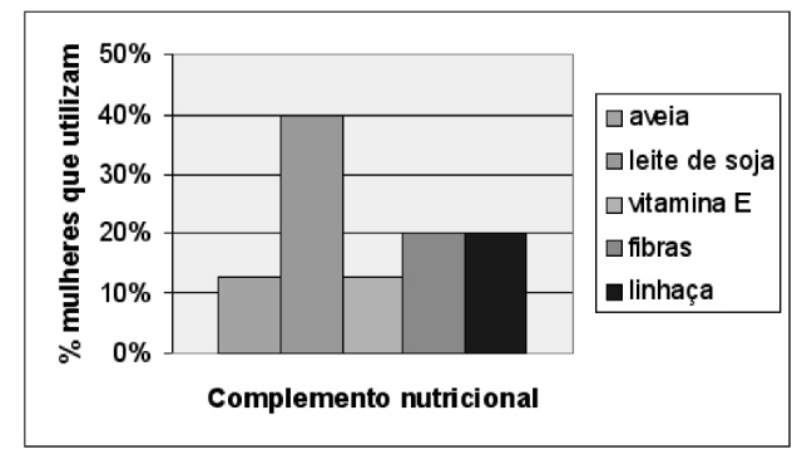

FIGURA 8 - PESQUISA DA DIETAALIMENTAR DO GRUPO

A microbiota intestinal, segundo MURRAY (1998), é necessária para as devidas metabolização e conjugação das isoflavonas para que elas possam ser absorvidas pela mucosa intestinal. A dieta alimentar do paciente é um dado importante para o farmacêutico que dispensa a isoflavona. A correta manipulação da isoflavona não basta para que a mulher que esteja fazendo uso tenha um bom resultado, é necessário e indispensável no serviço de Atenção Farmacêutica orientar para uma dieta rica em fibras a fim de garantir a ação da isoflavona.

\subsection{QUANTO AOS EXAMES LABORATORIAIS}

No grupo pesquisado $27 \%$ das mulheres nunca fizeram dosagem de hormônios sexuais, 13\% nunca fizeram dosagem de hormônios da tiróide e 13\% nunca fizeram dosagem de diabetes como está representado na FIGURA9.

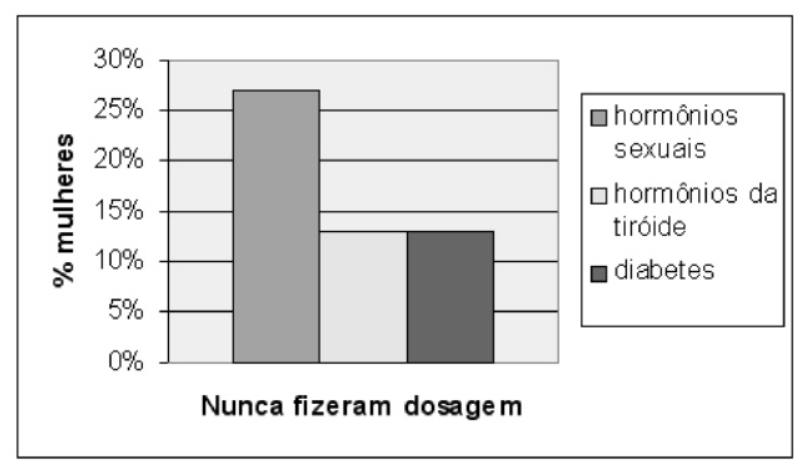

FIGURA 9 - EXAMES NUNCA REALIZADOS POR PARTE DAS MULHERES

Os dados acima demonstram precariedade no acompanhamento médico no que diz respeito aos hormônios sexuais uma vez que $47 \%$ das mulheres entrevistadas demonstraram diminuição do desejo sexual, aliado a $53 \%$ que apontou secura vaginal conforme já apresentado na TABELA 1. Ainda pode-se afirmar precariedade em relação à dosagem de hormônios tireoideanos $(13 \%)$, bem como aqueles relacionados ao diabetes $(13 \%)$.

Tal afirmação baseia-se em MARTINS (1996), onde o autor afirma ser o diagnóstico da síndrome menopausal essencialmente clínico, mas contudo torna-se imprescindível o acompanhamento da dosagem de hormônios sexuais. Cabe ressaltar 
que somente $6 \%$ das receitas se referem à especialidade médica ginecologia e $19 \%$ foram emitidas por clínicos gerais, talvez resulte disto o desinteresse pela dosagem de hormônios sexuais e tireoideanos.

\section{CONCLUSÃO}

A menopausa é um período do ciclo da vida feminina que pressupõe mudanças sistêmicas, que incluem desde sintomas físicos até desníveis biológicos (celulares químicos) que alteram todo o metabolismo, existindo a necessidade de avaliar a mulher neste estágio como um ser humano completo, considerando o perfil psicológico, 0 impacto dos sintomas no dia-a-dia e as doenças que podem estar associadas à faixa etária.

A aplicação de um instrumento de avaliação a um grupo de mulheres que se encontram na menopausa, permitiu a coleta de dados referente aos sintomas mais freqüentes, às doenças associadas e aos cuidados especiais (uso de hidratante/ filtro solar, nutrição, exercícios físicos e tabagismo). Os resultados encontrados para os cuidados especiais foram satisfatórios no que diz respeito ao uso de hidratante, ao uso de filtro solar e à prática de exercícios, mas vale a pena ressaltar que as mulheres participantes pertencem à classe média alta, a qual possui como tradição a preocupação com a estética e a beleza. A detecção de doenças associadas e de sintomas mais freqüentes, permite que o farmacêutico através de uma entrevista com a paciente aponte caminhos na busca de uma pesquisa clínica mais abrangente com especialidades mais dirigidas.

A avaliação das prescrições médicas e dos instrumentos de avaliação demonstraram que $89 \%$ das formulações prescritas foram cápsulas, destas $74 \%$ são isoflavonas, das quais $69 \%$ foram prescritas por dermatologistas. A dermatologia é uma especialidade médica que tem grande parte de suas prescrições direcionadas à farmácia de manipulação, mas um fato que deve ser considerado é a amplitude da dosagem da isoflavona que foi prescrita (10mg 160mg). Esse grande intervalo pressupõe que a prescrição não segue protocolos de tratamento e que os efeitos sistêmicos bem como suas interações medicamentosas podem não estar sendo considerados, havendo a necessidade de mais estudos a respeito dos efeitos e dosagem da isoflavona. $O$ alto percentual da prescrição da forma farmacêutica cápsulas, exige observação do farmacêutico no que se refere às peculiaridades de cada paciente, às associações de fármacos, ao fator de correção, à estabilidade e à compatibilidade dos fármacos, segundo à via de administração requerida e a forma farmacêutica associada.

A Atenção Farmacêutica promove um estreitamento no relacionamento do farmacêutico com o médico, por meio do paciente. Este por sua vez, pode dirigir-se ao médico já com o registro de sintomas associados, de medicamentos prescritos por outras especialidades médicas, da possibilidade de incompatibilidade farmacológica e do acompanhamento da farmacoterapia. O farmacêutico tendo posse dessas informações, pode inclusive sugerir ao médico alterações na posologia ou até mesmo, substituição da farmacoterapia quando o acompanhamento não detecta melhoras para a saúde do paciente ou compromete a qualidade de vida do mesmo. Assim, o farmacêutico tem seu reconhecimento não só por parte dos pacientes, mas sim, por outros profissionais da área de saúde, que juntos podem por meio de um trabalho 
multidisciplinar proporcionar um tratamento adequado com resultados terapêuticos satisfatórios e garantir a qualidade de vida dos pacientes. No entanto, para que o farmacêutico exercite a Atenção Farmacêutica, faz-se necessário uma capacitação profissional adequada por meio de uma formação acadêmica mais humanista e de aperfeiçoamentos na área.

\section{REFERÊNCIAS}

ABREU, V.; FRANCISCHETTI, E. A. Reposição Hormonal: controvérsias. Revista Brasileira de Medicina, abril 2001, vol. 25, no 04.

Accursio, C. S. C. Alterações de Pele na Terceira Idade. Revista Brasileira de Medicina, set. 2001, vol. 58, no 9, p. 646-658. Disponível em: $<$ http://www.cibersaude.com.br/revistas.asp?fase=r003\&id_matéria=1658> acesso em: 18/07/04.

ALDRIGHI, J. M.; ARAUJO, S. D. T. História Natural da Osteosporose na Mulher no Climatério. Revista Brasileira de Medicina., ago 1999, vol 56. Disponível em: $<$ http://www.cibersaude.com.br/revistas.asp?fase $=$ r003\&id materia $=559$ > Acesso em: 07/11/04.

ALVES, D. L.; SILVA, C. R. Fitohormônios: Abordagem Natural da Terapia Hormonal. 1 ed. São Paulo: Atheneu, 2003.

AMADO, T. C. F.; ARRUDA, I. K. Hipertensão Arterial no idoso e fatores de risco associados. Rev Bras Nutr Clin 2004, vol.19, no 2, p. 94-99.

BArona, M. I. Menopausia y Piel Parte I: Aspectos Fisiopatogénicos. Revista Colombiana de Menopausia, enero/ marzo 2003, vol. 09, no 01. Disponível em: <http://www.encolombia.com> Acesso em: 18/07/04.

Barona, M. I. Menopausia y Piel III. Revista Colombiana de Menopausia, abril/ junio 2003, vol. 9, no 02. Disponível em: <http://www.encolombia.com> Acesso em: $18 / 07 / 04$.

BELTZ, C. R.; ANTUNANO, S. P. G. Fitoestrógenos:Estado Actual. Revista Colombiana de Menopausa, enero/marzo 2003, vol. 09, no 01. Disponível em: <http://www.encolombia.com> Acesso em: 30/09/04.

BRASIL; Ministério da Saúde; Secretaria de Assistência à Saúde. Assistência ao climatério. Brasil, 1994.164p.

CARRERETTE, F. B.; DAMIAO, R. Incontinência Urinária na Mulher na Visão da Urologia. Incontinência Urinária, nov 1999, vol 56, no 11. disponível em: < www.cibersaude.com.br/revistas.asp?fase=r001\&id_=31 >Acesso em: 01/11/04.

CELESTINO, A. A. Etiopatogenia da Osteosporose. Revista Brasileira de Medicina, a g o $1999, \quad$ v o l $\quad 56 . \quad$ D i s p o n í v e l em:<http://www.cibersaude.com.br/revistas.asp?fase $=$ r003\&id_materia $=558>$ Acesso em: 07/11/04.

COBRA, M. Estratégias de Marketing de Serviços. 2 ed. São Paulo: Cobra Editora \& Marketing, 2001.

CONSELHO FEDERAL DE FARMÁCIA. Resolução n. 417 de 29 de setembro de 2004. Dispõe sobre o Código de Ética da Profissão Farmacêutica. Relator: Jaldo de Souza Santos, Diário Oficial da República Federativa do Brasil, Brasília, 29 de setembro de 2004.

COSTA-PAIVA, L.; HOROVITZ, A. P.; SANTOS, A. O. ; FONSECHI-CARVASAN, G. A. 
; PINTO-NETO, A. M. Prevalência de Osteoporose em Mulheres na Pósmenopausa e Associação com Fatores Clínicos e Reprodutivos. Rev. Bras. Ginecol. Obstet., Ago 2003, vol.25, no.7, p.507-512.

Deborah, J. L. Female Sexual Dysfunction. Mayo Clin Proc. 2002, vol. 77, p. 698-702. DemÉTrio, F. N. Saúde Mental da Mulher no Climatério. Revista Brasileira de Medicina Ginecologia e Obstetrícia, ago. 2000, v. 11, no. 2. Disponível em: http://www.cibersaude.com.br/revistas.asp?fase=r003\&id materia=112> Acesso em: 22/08/04.

FETROW, C. W.; AVILA, Juan R. Manual de Medicina Alternativa para o Profissional. Rio de Janeiro: Editora Guanabara Koogan, 2000.

FONSECA, A. M.; BAGNOLI, V. R.; CARDOSO, E. B.; ASSIS, J. S. Climatério: abordagem atual do diagnóstico e tratamento. Revista Brasileira de Medicina, set 200, vol. 57, no 09, p. 6570.

GALENA. Isoflavin Beta Glycine Max. L. (Mínimo $\mathbf{4 0 \%}$ de isoflavonas Totais) Fitoestrógenos da Soja. Informe Científico, set 2002, p. 1-8.

GUARISI, T.; PINTO-NETO, A. M.; OSIS, M. J. ; ORCESI, A.; LUCIA, P.;COSTA-PAIVA, H. S.; FAGUNDES, A. Procura de Serviço Médico por Mulheres com Incontinência Urinária. Rev. Bras. Ginecol. Obstet., ago 2001, vol 23, no 7, p. 439-443.

HAN, K. K.; SOARES JUNIOR, J. M. ;HAIDAR, M. H.; GIRÃO, M. J. B. C.; NUNES, M. G.;LIMA, G. R.; BARACAT, E. C. Efeitos dos Fitoestrógenos sobre Alguns Parâmetros Clínicos e Laboratoriais no Climatério. Revista Brasileira de Ginecologia e Obstetrícia, vol. 24, no 8, 2002, p. 547-552.

HANSEN JUNIOR, G. Marketing na Farmácia de Manipulação. Sp Eventos e Consultorias, Curitiba, 2004.

HEGG, R. Aspectos Práticos na Prevenção e Tratamento da Osteoporose. Revista Brasileira de Medicina, jun 2001, vol 58, no 6. Disponível em: < http://www.cibersaude.com.br/revistas.asp?fase=r003\&id_materia=1537>Acesso em: 07/11/04.

KOROLKOVAS, A.; FRANÇA, F. F. A. C. Dicionário Terapêutico Guanabara. Rio de Janeiro: Editora Guanabara Koogan, 2000.

MARCONI, M. A.; LAKATOS, E. M. Técnicas de Pesquisa: planejamento e execução de pesquisas, amostragens e técnicas de pesquisa, elaboração, análise e interpretação de dados.2 ed. São Paulo: Atlas, 1990.

MARTINS, A. A. Menopausa Sem Mistérios: as mais recentes descobertas. Rio de Janeiro: Rosa dos Tempos, 1996.

MELO, N. R.; GRINBAUM, M. L.; FERNANDES, C. E.; FERREIRA, J. A. S. TRH Bajas dosis hormonales. Revista Colombiana de Menopausia, julio/sept 2002, vol. 08, no 03. Disponível em: <http://www.encolombia.com> Acesso em: 18/07/04.

MENDOÇA, M.; CAMARGO, R. C.; FERREIA, R. C. ; SILVA, R. E. Tabagismo e sua Inter-relação com Doenças Ginecológicas. Jornal Brasileiro de Medicina, março 2004, vol 86, no 03, p 60 - 63.

MIGUEL, M. D.; ZANIN, S. M. W.; MIGUEL, O. G; ROZE, A. O.; OYAKAWA, C. N.; OLIVEIRA, A. B. de. O cotidiano das farmácias de manipulção. Revista Visão Acadêmica, jul/dez 2002, vol. 3, no 02.

MINISTÉRIO DAEDUCAÇÃO. Lei n. 9394, de 20 de dezembro de 1996. Dispõe sobre as Diretrizes e Base para a Regulamentação do Ensino. Relator: Paulo Renato Souza, Diário Oficial da República Federativa do Brasil, Brasília, 20 de dezembro de 1996. NEWALL, C.A.; ANDERSON, L. A.; PHILLIPSON, D. J. Plantas Medicinais Guia 
para Profissional de Saúde. São Paulo: Editorial Premier, 2002.

OSIO, G. A. Transtornos del sueno durante la menopausia. Revista Colombiana de Menopausa, enero/abril 2001, vol. 07, no 01. Disponível em : $<$ http://www.encolombia.com>Acesso em: 25/11/04.

PAULOS, C. Atenção Farmacêutica: Um Desafio da Profissão Farmacêutica. Revista Racine, março/ abril 2002, vol 67, ano XII, p. 42-48.

PCCA BRASIL. Novo Alerta Sobre o Risco de Reposição Hormonal. O Globo RJ. Disponível em: <www.pccabrasil.com.br/frame.htm>Acesso em: 03/03/04.

RANG H. P.; RITTER J. M.; DALE M. M. Farmacologia. 3 ed. Rio de Janeiro: Editora Guanabara Koogan S.A., 1997.

ROSANO, G.; MERCURIO, G.; ONORATI, D.; VITALE, C.; FINI, M. Las progestinas anulan el efecto protector de los estrogenos sobre el riesgo cardiovascular? Revista Colombiana de Menopausia, mayo/ agosto 2001, vol. 07, no 02. Disponível em: <http://www.encolombia.com> Acesso em: 20/11/04.

ROSANO, G.; MERCURIO, G.; ONORATI, D.; VITALE, C.; FINI, M. Terapia de Reemplazo Hormonal y cardioproteccion en las mujeres menopausicas: estudios clinicos y epidemiológicos. Revista Colombiana de Menopausia, enero/marzo 2002, vol. 08, no 01. Disponível em: <http://www.encolombia.com> Acesso em: 25/10/04.

ROSSI, P. de ; RIBEIRO, R. M.; MELO, N. R. de; PINOTTI, J. A. Distúrbios Urogenitais no Climatério. Revista Brasileira de Medicina, Jun 01, vol. 58, $\mathrm{n}^{\circ} 06$.

RUIZ-LARREA, M. B.; RUIZ-SANZ, J. I. Revisión Temática Fitoestrógenos y Salud. Antioxidantes y Calidad de Vida, outubro 2003. Disponível em: $<$ www.antioxidantes.com.ar/12/home2.htm> Acesso em: 23/12/04.

SAMARA, B. S.; BARROS, J. C. Pesquisa de Marketing Conceito e Metodologia. 2 ed. São Paulo:Makron Books, 1997.

SANDRON, C. A. Atenção Farmacêutica e Farmacovigilância. Revista Racine, março/abril 2002, vol67, ano XII, p. 20-24.

SCHULZ, V.; HANSEL, R.; TYLER, V. E. Um Guia de Fitoterapia Para as Ciências da Saúde. 4 ed. São Paulo: Editora Mamole,2002.

SIMMONS, C. Terapia de Reposição Hormonal Feminina e Masculina com Hormônios Bioidênticos. PCCA, Brasil, 2003.

SOUZA, H. G. de. Assistência Farmacêutical Atenção Farmacêutica: Uma Experiência na Farmácia com Manipulação. Revista Racine, março/ abril 2002, vol 67, ano XII, p. 30-36.

Steiner, D. Dermatologia da Mulher no Climatério. Revista Brasileira de Medicina Ginecologia e Obstetrícia, ago. 2000, vol. 11, no. 2. Disponível em: $<$ http://www.cibersaude.com.br/revistas.asp?fase=r003\&id_materia=112 > Acesso em: 18/07/04

TABARES, G. G.; ALFARO, S. P. Nutricion y menopausia. Revista Colombiana de Menopausia, abril/ junio 2002, vol. 08, no 02. Disponível em: $<$ http://www.encolombia.com> Acesso em: 19/07/04.

ZARATE, A.; BASURTO, L. La disfunción tiroidea es frecuente en la mujer. Revista Colombiana de Menopausia, enero/ abril 2001, vol. 07, no 01. Disponível em: <http://www.encolombia.com> Acesso em: 30/07/04. 